\title{
Proving and Extending Greub-Reinboldt Inequality Using the Two Nonzero Component Lemma
}

\author{
Morteza Seddighin \\ Indiana University East, Richmond, IN 47374, USA \\ Email: mseddigh@iue.edu
}

Received 12 May 2014; revised 2 June 2014; accepted 9 June 2014

Copyright (C) 2014 by author and Scientific Research Publishing Inc.

This work is licensed under the Creative Commons Attribution International License (CC BY). http://creativecommons.org/licenses/by/4.0/

\section{(c) (i) Open Access}

\begin{abstract}
We will use the author's Two Nonzero Component Lemma to give a new proof for the GreubReinboldt Inequality. This method has the advantage of showing exactly when the inequality becomes equality. It also provides information about vectors for which the inequality becomes equality. Furthermore, using the Two Nonzero Component Lemma, we will generalize GreubReinboldt Inequality to operators on infinite dimensional separable Hilbert spaces.
\end{abstract}

\section{Keywords}

\section{Greub-Reinboldt Inequality, Two Nonzero Component Lemma}

\section{Introduction}

Many authors have established Kantorovich inequality and its generalizations such as Greub-Reinboldt Inequality by variational methods. In a variational approach, one differentiates the functional involved to arrive at an "Euler Equation" and then solves the Euler Equating to obtain the minimizing or maximizing vectors of the functional involved. Solving these Euler Equations is tedious and generally provides little information (see [1], subsection 4.4 for an example of this method). Others have established Kantorovich-type inequalities for positive operators by going through a two-step process which consists of first computing upper bounds for suitable functions on intervals containing the spectrum of suitable matrix and then applying the standard operational calculus to that matrix (see [2]) for an example of this method. This method, which we refer to as "the operational calculus method", has the following two limitations:

First, it does not provide any information about vectors for which the established inequalities become equalities. Second, the operational calculus method is futile in extending Kantorovich-type inequalities to operators on an infinite dimensional Hilbert space. A number of Kantorovich-type inequalities are discussed in [3]. 
In this paper we use the author's Two Nonzero Component Lemma to prove, improve and extend matrix form of Greub-Reinboldt Inequality.

\section{The Two Nonzero Component Lemma}

In his investigation on problems of antieigenvalue theory the author has discovered a useful lemma which he calls it the Two Nonzero Component Lemma (see [4]-[6]). Although this Lemma is implicitly used in all of the papers just cited, it was not until 2008 that he stated a formal description of the Lemma in his paper titled, "Antieigenvalue Techniques in Statistics". Below is the statement of the lemma. For the proof of the lemma please see the author's work cited above.

Lemma 1 (The Two Nonzero Component Lemma) Let $l_{1}^{+}$be the set of all sequences with nonnegative terms in the Banach Space $l_{1}$. That is, let

$$
l_{1}^{+}=\left\{\mathbf{t}=\left(t_{i}\right) \in l_{1}, t_{i} \geq 0\right\} .
$$

Let

$$
F\left(x_{1}, x_{2}, \cdots, x_{m}\right)
$$

be a function from $R^{m}$ to $R$. Assume $g_{k}(\mathbf{t})=\sum c_{i}^{k} t_{i}$ for $\left(c_{i}^{k}\right) \in l_{1}^{+}, \mathbf{t} \in l_{1}^{+}$, and $1 \leq k \leq m$. Then the minimizing vectors for the function

$$
F\left(g_{1}(\mathbf{t}), g_{2}(\mathbf{t}), \cdots, g_{m}(\mathbf{t})\right)
$$

on the convex set $C=\left\{\left(t_{i}\right) \in l_{1}: \sum t_{i}=1\right\}$ have at most two nonzero components.

What make the proof of the Lemma possible are the following two facts: First, the convexity of the set

$$
C=\left\{\left(t_{i}\right) \in l_{1}^{+}: \sum t_{i}=1\right\} .
$$

Second, a special property that the functions

$$
F\left(g_{1}(\mathbf{t}), g_{2}(\mathbf{t}), \cdots, g_{m}(\mathbf{t})\right)
$$

involved possess. If we set

$$
D\left(t_{1}, t_{2}, t_{3}, \cdots\right)=F\left(g_{1}(\mathbf{t}), g_{2}(\mathbf{t}), \cdots, g_{m}(\mathbf{t})\right)
$$

then all restrictions of the form

$$
D\left(t_{1}, t_{2}, \cdots, t_{i-1}, 0, t_{i+1}, \cdots\right)
$$

of

$$
D\left(t_{1}, t_{2}, t_{3}, \cdots\right)
$$

have the same algebraic form as $D\left(t_{1}, t_{2}, t_{3}, \cdots\right)$ itself. For example if

$$
D\left(t_{1}, t_{2}, \cdots, t_{n}\right)=\frac{\beta_{1} t_{1}+\beta_{2} t_{2}+\cdots+\beta_{n} t_{n}}{\left|\lambda_{1}\right|^{2} t_{1}+\left|\lambda_{2}\right|^{2} t_{2}+\cdots+\left|\lambda_{3}\right|^{2} t_{3}},
$$

then we have

$$
D\left(0, t_{2}, \cdots, t_{n}\right)=\frac{\beta_{2} t_{2}+\cdots+\beta_{n} t_{n}}{\left|\lambda_{2}\right|^{2} t_{2}+\cdots+\left|\lambda_{3}\right|^{2} t_{3}}
$$

which has the same algebraic form as

$$
D\left(t_{1}, t_{2}, \cdots, t_{n}\right)=\frac{\beta_{1} t_{1}+\beta_{2} t_{2}+\cdots+\beta_{n} t_{n}}{\left|\lambda_{1}\right|^{2} t_{1}+\left|\lambda_{2}\right|^{2} t_{2}+\cdots+\left|\lambda_{3}\right|^{2} t_{3}} .
$$

Indeed, for any $j, 1 \leq j<n$; all restrictions of the function 


$$
D\left(t_{1}, t_{2}, \cdots, t_{n}\right)=\frac{\beta_{1} t_{1}+\beta_{2} t_{2}+\cdots+\beta_{n} t_{n}}{\left|\lambda_{1}\right|^{2} t_{1}+\left|\lambda_{2}\right|^{2} t_{2}+\cdots+\left|\lambda_{3}\right|^{2} t_{3}}
$$

obtained by setting an arbitrary set of $j$ components of $D\left(t_{1}, t_{2}, \cdots, t_{n}\right)$ equal to zeros have the same algebraic form as $D\left(t_{1}, t_{2}, \cdots, t_{n}\right)$. Obviously, not all functions have this property. For instance, for the function $G\left(t_{1}, t_{2}\right)=2 t_{1}+t_{1} t_{2}, G\left(t_{1}, 0\right)=2 t_{1}$, which does not have the same algebraic form as $G\left(t_{1}, t_{2}\right)$.

\section{Greub-Reinboldt Inequality}

Let $x=\left(x_{1}, x_{2}, \cdots, x_{n}\right)$ and $y=\left(y_{1}, y_{2}, \cdots, y_{n}\right)$ be two real $n$-tuples. Suppose that $m_{1}, M_{1}, m_{2}, M_{2}$ are constants such that $m_{1} \leq x_{i} \leq M_{1}$ and $m_{2} \leq y_{i} \leq M_{2}$. Then, for $w_{i}>0$ we have

$$
\sum_{i=1}^{n} w_{i} x_{i}^{2} \sum_{i=1}^{n} w_{i} y_{i}^{2} \leq \frac{\left(M_{1} M_{2}+m_{1} m_{2}\right)^{2}}{4 m_{1} m_{2} M_{1} M_{2}}\left(\sum_{i=1}^{n} w_{i} x_{i} y_{i}\right)^{2} \text {. }
$$

A slightly different form of the above inequality was proved by J. W. S. Cassels in 1951 (see Appendix 1 of [7]). In the following section we provide a proof for the matrix form of Greub-Reinboldt Inequality based on the Two Nonzero Component Lemma. The proof is completely different than the proofs given by others, including Greub and Reinboldt themselves (see [8]). This proof has the advantage of providing information about when the inequality becomes equality and gives information about vectors which make the inequality equality. Furthermore, as we will discuss in the Section 5, our method will indeed extend the Greub-Reinboldt Inequality to operators on an infinite dimensional Hilbert space.

\section{The Matrix Form of Greub-Reinboldt Inequality}

Theorem 2 Let $S$ and $T$ be two commuting positive operators with eigenvalues $\left\{\alpha_{i}\right\}_{i=1}^{n}$ and $\left\{b_{i}\right\}_{i=1}^{n}$ respectively. Assume

$$
m_{1} \leq \alpha_{1} \leq \cdots \leq \alpha_{n} \leq M_{1}
$$

and

$$
m_{2} \leq \beta_{1} \leq \cdots \leq \beta_{n} \leq M_{2} .
$$

Also assume $S$ and $T$ are diagonalized with diagonal elements $\left\{\alpha_{i}\right\}_{i=1}^{n}$ and $\left\{\beta_{i}\right\}_{i=1}^{n}$ respectively, then

$$
(T x, T x)(S x, S x) \leq \frac{\left(M_{1} M_{2}+m_{1} m_{2}\right)^{2}}{4 m_{1} m_{2} M_{1} M_{2}}(T x, S x)^{2}
$$

for every vector $x$. In this, case if $x$ is any unit vector which makes the inequality (15) an equality then we have

$$
\left\|x_{1}\right\|^{2}=\frac{\alpha_{n} \beta_{n}}{\alpha_{1} \beta_{1}+\alpha_{n} \beta_{n}}
$$

and

$$
\left\|x_{n}\right\|^{2}=\frac{\alpha \beta_{1}}{\alpha_{1} \beta_{1}+\alpha_{n} \beta_{n}}
$$

and

$$
x_{i}=0 \text { if } 1<i<n
$$

where $x_{i}$ is the projection of $x$ on the eigenspace corresponding to eigenvalue $\alpha_{i}$.

Proof. Without loss of generality we can assume $\|x\|=1$. Consider the functional

$$
\frac{(T x, T x)(S x, S x)}{(T x, S x)^{2}} \text {. }
$$


(19) can be written as

$$
\frac{\|T x\|^{2}\|S x\|^{2}}{(T x, S x)^{2}}
$$

The reciprocal of (20) is

$$
\frac{(T x, S x)^{2}}{\|T x\|^{2}\|S x\|^{2}}
$$

The square root of (21) is

$$
\frac{(T x, S x)}{\|T x\|\|S x\|}
$$

To prove (15) we first find

$$
\inf _{\|x\|=1} \frac{(S T x, x)}{\|T x\|\|S x\|} .
$$

Since $S$ is invertible, by a change of variable we have

$$
\inf _{\|x\|=1} \frac{(S T x, x)}{\|T x\|\|S x\|}=\inf _{\|x\|=1} \frac{\left(T S^{-1} x, x\right)}{\left\|T S^{-1} x\right\|\|x\|} .
$$

By the spectral mapping theorem the inf on the right hand side of (24) can be represented as

$$
\inf \frac{\sum_{i=1}^{n} \lambda_{i}\left\|x_{i}\right\|^{2}}{\sqrt{\sum_{i=1}^{n} \lambda_{i}^{2}\left\|x_{i}\right\|^{2}}}
$$

over the set

$$
\sum_{i=1}^{n}\left\|x_{i}\right\|^{2}=1
$$

where $\left\{\lambda_{i}\right\}_{i=1}^{\infty}$ is the set of eigenvalues of $T S^{-1}$. The fact that $S$ and $T$ commute implies that

$$
\lambda_{i}=\alpha_{i}^{-1} \beta_{i}
$$

for $1 \leq i \leq n$ and

$$
m=M_{1}^{-1} m_{2} \text { and } M=m_{1}^{-1} M_{2}
$$

where

$$
m=\min \left\{\lambda_{i}\right\}_{i=1}^{n}
$$

and

$$
M=\max \left\{\lambda_{i}\right\}_{i=1}^{n}
$$

If we set $\left\|x_{i}\right\|^{2}=t_{i}$ the problem is reduced to finding

$$
\inf \frac{\sum_{i=1}^{n} \lambda_{i} t_{i}}{\sqrt{\sum_{i=1}^{n} \lambda_{i}^{2} t_{i}}}
$$


over

$$
\sum_{i=1}^{n} t_{i}=1
$$

By the Two Nonzero Component Lemma we need to look at

$$
\inf \frac{\lambda_{i} t_{i}+\lambda_{j} t_{j}}{\sqrt{\lambda_{i}^{2} t_{i}+\lambda_{i}^{2} t_{i}}}
$$

over the convex set

$$
t_{i}+t_{j}=1
$$

for pairs of $i$ and $j$. Notice that since the expression in (33) is positive, for simplicity, we can first compute the infimum of the square of that expression on the convex set (34) and then take square root of the result Therefore, the problem is now reduced to finding

$$
\inf \frac{\left(\lambda_{i} t_{i}+\lambda_{j} t_{j}\right)^{2}}{\lambda_{i}^{2} t_{i}+\lambda_{i}^{2} t_{i}}
$$

on (34). By substituting $t_{j}=1-t_{i}$ in (35) the problem is now reduced to finding

$$
\inf \frac{\left(\lambda_{i} t_{i}+\lambda_{j}\left(1-t_{i}\right)\right)^{2}}{\lambda_{i}^{2} t_{i}+\lambda_{i}^{2}\left(1-t_{i}\right)}
$$

for $0 \leq t_{i} \leq 1$. To find (36), simply differentiate the expression in (36) and set its derivative with respect to $t_{i}$ equal to zero (we omit the straight forward computations). The expression in (36) is minimized when

$$
t_{i}=\frac{\lambda_{j}}{\lambda_{i}+\lambda_{j}} .
$$

Substituting this value of $t_{i}$ in (34) and the expression in (36) gives us

$$
t_{j}=\frac{\lambda_{i}}{\lambda_{i}+\lambda_{j}}
$$

and

$$
\frac{\left(\lambda_{i} t_{i}+\lambda_{j}\left(1-t_{i}\right)\right)^{2}}{\lambda_{i}^{2} t_{i}+\lambda_{i}^{2}\left(1-t_{i}\right)}=\frac{4 \lambda_{i} \lambda_{j}}{\left(\lambda_{i}+\lambda_{j}\right)^{2}} .
$$

Hence

$$
\inf \frac{\lambda_{i} t_{i}+\lambda_{j} t_{j}}{\sqrt{\lambda_{i}^{2} t_{i}+\lambda_{i}^{2} t_{i}}}=\frac{2 \sqrt{\lambda_{i} \lambda_{j}}}{\lambda_{i}+\lambda_{j}}
$$

and the inf in (39) is attained at

$$
t_{i}=\frac{\lambda_{j}}{\lambda_{i}+\lambda_{j}}
$$

and

$$
t_{j}=\frac{\lambda_{i}}{\lambda_{i}+\lambda_{j}} .
$$

Assume that $i<j$. Now we show that we must have $i=1$ and $j=n$. To prove this we must show

$$
\frac{2 \sqrt{\lambda_{1 i} \lambda_{n}}}{\lambda_{1}+\lambda_{n}} \leq \frac{2 \sqrt{\lambda_{i} \lambda_{j}}}{\lambda_{i}+\lambda_{j}}
$$


for $1 \leq i \leq n$ and $1 \leq i \leq n$. Squaring both sides of (42) gives us

$$
\frac{4 \lambda_{1} \lambda_{n}}{\left(\lambda_{1}+\lambda_{n}\right)^{2}} \leq \frac{4 \lambda_{i} \lambda_{j}}{\left(\lambda_{i}+\lambda_{j}\right)^{2}}
$$

Thus instead of proving inequality (42) we can prove inequality (43). Let $u=\frac{\lambda_{n}}{\lambda_{1}}$ and $v=\frac{\lambda_{j}}{\lambda_{i}}$. It is obvious that

$$
1 \leq y \leq x .
$$

If we substitute $\lambda_{n}=u \lambda_{1}$ and $\lambda_{j}=v \lambda_{i}$ in (43) we get

$$
\frac{4 u \lambda_{1}^{2}}{\lambda_{1}^{2}(1+u)^{2}} \leq \frac{4 v \lambda_{i}^{2}}{\lambda_{i}^{2}(1+v)^{2}}
$$

which is equivalent to

$$
\frac{4 u}{(1+u)^{2}} \leq \frac{4 v}{(1+v)^{2}} .
$$

Hence proving inequality (42) is reduced to proving inequality (46). To prove (46), note that based on (44)

$$
\frac{4 u}{(1+u)^{2}}-\frac{4 v}{(1+v)^{2}}=\frac{(u-v)(1-u v)}{(u+1)^{2}(v+1)^{2}} \leq 0 .
$$

Therefore, we must have

$$
\inf _{\|x\|=1} \frac{(S T x, x)}{\|T x\|\|S x\|}=\frac{2 \sqrt{\lambda_{1 i} \lambda_{n}}}{\lambda_{1}+\lambda_{n}} .
$$

The inequality

$$
\frac{2 \sqrt{\lambda_{1} \lambda_{n}}}{\lambda_{1}+\lambda_{n}} \geq \frac{2 \sqrt{m M}}{n+M}
$$

can be proved the same way we just proved (42). Hence we have

$$
\frac{(S T x, x)^{2}}{\|T x\|^{2}\|S x\|^{2}} \geq \frac{4 M_{1}^{-1} m_{2} m_{1}^{-1} M_{2}}{\left(M_{1}^{-1} m_{2}+{ }_{2} m_{1}^{-1} M_{2}\right)^{2}} .
$$

The right side of 50 is simplified to

$$
\frac{4 m_{1} m_{2} M_{1} M_{2}}{\left(M_{1} M_{2}+m_{1} m_{2}\right)^{2}}
$$

Thus (50) becomes

$$
\frac{(S T x, x)^{2}}{\|T x\|^{2}\|S x\|^{2}} \geq \frac{4 m_{1} m_{2} M_{1} M_{2}}{\left(M_{1} M_{2}+m_{1} m_{2}\right)^{2}} .
$$

Finally (50) is equivalent to

$$
(T x, T x)(S x, S x) \leq \frac{\left(M_{1} M_{2}+m_{1} m_{2}\right)^{2}}{4 m_{1} m_{2} M_{1} M_{2}}(T x, S x)^{2} .
$$

\section{Generalizing Greub-Reinboldt Inequality to Operators on a Separable Hilbert Space}

There are many proofs for Greub-Reinboldt Inequality in the literature. A significant advantage of proving 
Greub-Reinboldt Inequality by The Two Nonzero Component Lemma is that we can generalize this inequality to the case of positive operators $S$ and $T$ on an separable infinite dimensional separable Hilbert space. This is because, as the statement of the Two Nonzero Component Lemma shows, this lemma is also when the functions $g_{k}(\mathbf{t})=\sum c_{i}^{k} t_{i}$ infinite linear combinations of $t_{1}, t_{2}, t_{3}, \cdots$ Thus we can replace finite summations in (25), (26), (31), (32) with infinite sums and the arguments made in this paper remain valid. However, in this case it seems difficult to the pinpoint the exact pair of $i$ and $j$ for which the projections $x_{i}$ and $x_{j}$ of minimizing unit vectors are nonzero.

Theorem 3 Let $S$ and $T$ be two commuting positive operators on a separable Hilbert space such that $\sigma(S) \subseteq\left[m_{1}, M_{1}\right]$ and $\sigma(T) \subseteq \sigma(T) \subseteq\left[m_{2}, M_{2}\right]$ where $\sigma(S)$ and $\sigma(T)$ represent the spectrums of $S$ and $T$ respectively, then

$$
(T x, T x)(S x, S x) \leq \frac{\left(M_{1} M_{2}+m_{1} m_{2}\right)^{2}}{4 m_{1} m_{2} M_{1} M_{2}}(T x, S x)^{2}
$$

for any vector $x$. In this, case if $x$ is any unit vector which makes the inequality (54) an equality then there exist a pair of $i$ and $j$ such that

$$
\left\|x_{i}\right\|^{2}=\frac{\alpha_{j} \beta_{j}}{\alpha_{i} \beta_{i}+\alpha_{j} \beta_{j}}
$$

and

$$
\left\|x_{j}\right\|^{2}=\frac{\alpha_{i} \beta_{i}}{\alpha_{i} \beta_{i}+\alpha_{j} \beta_{j}}
$$

and

$$
x_{k}=0 \text { if } k \neq i \text { and } k \neq j
$$

where $x_{i}$ is the projection of $x$ on the eigenspace corresponding to eigenvalue $\alpha_{i}$.

There are other generalizations of Greub-Reinboldt Inequality. For example in ([9]) Gustafson extends this inequality to pair noncommuting positive matrices $A$ and $B$. However, he replaces the standard norm of the Hilbert space with the norm relative to $B$.

Conclusion 4 The Two Nonzero Component Lemma provides an effective way of proving the GreubReinboldt Inequality and extending it to positive operators on separable infinite dimensional Hilbert spaces. The author has also utilized this lemma to prove other Kantorovich-type inequalities. Please see ([4]-[6] [10] [11]).

\section{References}

[1] Gustafson, K. (2001) Forty Years of Antieigenvalue Theory and Applications. Numerical Linear Algebra with Applications, 01, 1-10.

[2] Kantorovich, L. (1948) Functional Analysis and Applied Mathematics. Uspekhi Matematicheskikh Nauk, 3, 89-185.

[3] Gustafson, K. and Rao, D. (1997) Numerical Range. Springer, Berlin. http://dx.doi.org/10.1007/978-1-4613-8498-4

[4] Gustafson, K. and Seddighin, M. (1989) Antieigenvalue Bounds. Journal of Mathematical Analysis and Applications, 143, 327-340. http://dx.doi.org/10.1016/0022-247X(89)90044-9

[5] Seddighin, M. (2002) Antieigenvalues and Total Antieigenvalues of Normal Operators. Journal of Mathematical Analysis and Applications, 274, 239-254. http://dx.doi.org/10.1016/S0022-247X(02)00295-0

[6] Seddighin, M. (2009) Antieigenvalue Techniques in Statistics. Linear Algebra and Its Applications, 430, $2566-2580$. http://dx.doi.org/10.1016/j.laa.2008.05.007

[7] Watson, G.S. (1955) Serial Correlation in Regression Analysis I. Biometrika, 42, 327-342. http://dx.doi.org/10.1093/biomet/42.3-4.327

[8] Greub, W. and Rheinboldt, W. (1959) On a Generalisation of an Inequality of L.V. Kantorovich. Proceedings of the American Mathematical Society, 10, 407-415. http://dx.doi.org/10.1090/S0002-9939-1959-0105028-3

[9] Gustafson, K. (2004) Interaction Antieigenvalues. Journal of Mathematical Analysis and Applications, 299, $179-185$. http://dx.doi.org/10.1016/j.jmaa.2004.06.012

[10] Seddighin, M. and Gustafson, K. (2005) On the Eigenvalues Which Express Antieigenvalues. International Journal of 
Mathematics and Mathematical Sciences, 2005, 1543-1554. http://dx.doi.org/10.1155/IJMMS.2005.1543

[11] Gustafson, K. and Seddighin, M. (2010) Slant Antieigenvalues and Slant Antieigenvectors of Operators. Journal of Linear Algebra and Applications, 432, 1348-1362. http://dx.doi.org/10.1016/j.laa.2009.11.001 